\title{
Tilted Convective Plumes in Numerical Experiments
}

\author{
A. Wirth \& B. Barnier \\ achim.wirth@hmg.inpg.fr \\ $L E G I$ \\ BP 53, 38041 Grenoble Cedex 9 \\ France
}

\begin{abstract}
The dynamics of a single convective plume in an isothermal ocean is investigated by numerically integrating the three dimensional Boussinesq equations. Our study emphasizes on the important consequences of a non-vanishing angle between the axis of rotation and the buoyancy force (gravity). Experiments are performed for four different values of the angle corresponding to open-ocean convection at latitude: $90^{\circ} \mathrm{N}, 60^{\circ} \mathrm{N}, 45^{\circ} \mathrm{N}$ and $0^{\circ} \mathrm{N}$.

We show that the horizontal component of the rotation vector leads to qualitative and quantitative changes in the convective dynamics of a single plume. Plume structures are aligned along the axis of rotation rather than the direction of gravity (tilted convection), the vertical velocity of the plume is reduced, and the mixing is enhanced by the horizontal component of the rotation vector. These results suggest, that in future parametrisations of ocean convection the effect of the horizontal component of the rotation vector should be included.
\end{abstract}

Key words: Geophysical fluid dynamics, ocean convection, traditional approximation

PACS: 65N35, 76M22, 76R05, 86A05

\section{Introduction}

The important role of deep convection on the overturning circulation at global scale is now well established ant its impact on the climat dynamics recognized (Willebrand et al., 2000). Deep convection is also a principal component of the carbon cycle and has a strong influence on the biological dynamics in parts of the worlds ocean (Wood et al., 1999).

Preprint submitted to Elsevier Science $\quad 7$ March 2005 
Convection in the world ocean is a highly intermittent process in space and time. Convective regions, also called convective chimneys, typically span a few hundreds of kilometers in the horizontal directions. The actual convection process, that is, the vertical exchange and mixing of water masses only takes a few days. During this time the convective chimney is composed of a large number of convective plumes measuring up to one kilometer in the horizontal directions (Schott and Leaman 1991). The downward transport of the heavy water within the plumes is almost perfectly compensated by an upward transport in between the plumes (Send and Marshall 1995). The plumes are the coherent structures governing the convective dynamics. A detailed understanding of the convection process is thus not conceivable without a detailed understanding of the plume dynamics. For a review on open-ocean convection we refer the reader to Killworth (1983) and Marshall and Schott (1999). The plumes can indeed be seen as the "atoms of convection" which, however can not be explicitely resolved in the numerical models of the ocean circulation at basin scale. Their impact on the resolved dynamics has to be parametrized in such models. Efficient parameterizations are and will be key to the model developpement. Indeed, the rapid advancement of numerical ocean models and their improvement asks for an increasing accuracy in the representation of several phenomena. Current parameterisations of convection used in state of the art numerical models are inspired by the physics of the convection process and respond to the necessity of removing unstable stratifications in a numerical ocean. Improving parameterisation of the convection process does require a detailled understanding of the dynamics and the important processes and parameters involved. In this paper we demonstrate, that the "traditional approximation", that is, neglecting the horizontal component of the rotation vector, is not appropriate when considering the deep convection processes, even at higher lattitudes.

Convective plumes in an isothermal ocean are investigated by numerically integrating the three dimensional Boussinesq equations. The effects of rotation on the plume dynamics are considered. We not only vary the magnitude of the rotation vector but also its direction. More precisely, in the majority of numerical calculations considering ocean dynamics, the traditional approximation is employed which completely neglects the horizontal component of the rotation vector. Rotation is thus supposed to be colinear with gravity (see e.g. Marshall et al., 1997, for a discussion on the traditional approximation in an oceanic context). The traditional approximation may be justified in instances where vertical velocities are small compared to their horizontal counterparts, that is, when non-hydrostatic terms can be neglected. When, however, the non-hydrostatic terms are essential for the dynamics, as in the case of convection, the traditional approximation has to be relaxed, especially in regions of the world ocean of not too high latitudes. For the here presented numerical investigation we choose an inclination of the rotation vector corresponding to open-ocean convection at latitudes: $90^{\circ} \mathrm{N}, 60^{\circ} \mathrm{N}, 45^{\circ} \mathrm{N}$ and $0^{\circ} \mathrm{N}$ a case of no 
rotation is added for the completeness of the dynamical picture. The angles of $45^{\circ} \mathrm{N}$ and $60^{\circ} \mathrm{N}$ correspond to the Gulf of Lions and the Labrador Sea, respectively, two major convection sites of the world oceans (Killworth 1983, Schott and Marshall 1999). The angle of $90^{\circ} \mathrm{N}$, the North Pole where gravity and rotation are aligned, presents the "traditional" convection case. Most Ocean General Circulation Models (OGCMS) use the traditional approximation.

While preparing this manuscript the work by Sheremet 2004, was brought to our attention which clearly demonstrates the influence of a finite angle between the buoyancy and the axis of rotation in laboratory experiments. These pioneering laboratory experiments by V. Sheremet determine not only the influence of the axis of rotation on the direction of convection but also present a theoretical discussion of the phenomena. Our here presented work can indeed be seen as the numerical supplement to the laboratory experiments of Sheremet 2004, which present a fortuitous possibility to validate our calculations. To determine the influence of a finite angle between the axis of rotation and the direction of gravity we, however, performed calculations for four different angles. Following the work by Sheremet 2004 we also use the term "tilted" to describe the convection in instances where the axis and rotation and the buoyancy force are not colinear.

\section{Model Description}

The mathematical model of the here presented ocean convection experiment are the Boussinesq equations (eq. 1) of an incompressible flow (eq. 2) in a rotating frame, supplemented by boundary conditions. The flow field is given by $\mathbf{u}$ and the scalar (temperature) field by $T$. The buoyant scalar is transported by the flow (3). The source term $S$ insures the prescribed heat fluxes through the upper boundary and the equation of state is linear with the expansion coefficient $\alpha$. The ocean surface is modeled by a free-slip rigid-lid boundary condition, while the ocean floor is modeled by a no-slip boundary condition. In the horizontal directions periodic boundary conditions are employed.

$$
\begin{aligned}
\partial_{t} \mathbf{u}+\mathbf{u} \cdot \nabla \mathbf{u}+2 \Omega \times \mathbf{u}+\nabla P & =\alpha g T \mathbf{e}_{\perp}+\nu \nabla^{2} \mathbf{u} \\
\nabla \cdot \mathbf{u} & =0 \\
\partial_{t} T+\mathbf{u} \cdot \nabla T & =\kappa \nabla^{2} T+S .
\end{aligned}
$$

The mathematical model is solved numerically using a pseudo-spectral scheme entirely based on Fourier expansion. The boundary conditions are implemented using a technique inspired by the immersed boundary condition (see Peskin 1977 and Goldstein et al., 1993). For a detailed discussion on the model 
and the new boundary technique we refer the reader to Wirth 2004. Our model will forth-worth be called HAROMOD (HARmonic Ocean MODel).

The coherent structures dominating the convective dynamics are known to have comparable horizontal and vertical scales. A fact that has to be reflected in the aspect ratio of the numerical grid, which is unity in all the calculations presented here. We also choose the friction coefficients equal in the horizontal and vertical direction and the Prandtl number is unity $(\kappa=\nu)$ in all calculations.

The area of integration spans $2 \mathrm{~km} \times 2 \mathrm{~km}$ in the horizontal directions and $3 \mathrm{~km}$ in the vertical. The friction coefficients are, $\nu=\kappa=.1 \mathrm{~m}^{2} / \mathrm{s}$ the expansion coefficient $\alpha=2 . \times 10^{-4} K^{-1}$, gravity is $g=9.81 \mathrm{~m} / \mathrm{s}^{2}$, density $\rho=1000 \mathrm{~kg} / \mathrm{m}^{3}$ and the specific heat capacity $c=3900 \mathrm{~J} /(\mathrm{kg} \mathrm{K})$. The temperature in the upper 40 meters is perfectly mixed, that is, the temperature is homogenized in this layer at every time-step. The ocean is cooled in a circular region attached to the surface, using a Gaussian profile given by:

$$
H=1.28 \frac{W}{m^{3}} \exp \left(-\frac{x^{2}+y^{2}}{(200 m)^{2}}-\frac{z^{2}}{(70 m)^{2}}\right),
$$

leading to a surface heat flux of

$$
H_{s}=80 \frac{W}{m^{2}} \exp \left(-\frac{x^{2}+y^{2}}{(200 m)^{2}}\right)
$$

The total heating is thus $P=\int_{R^{3}} H d V=1 . \times 10^{7} \mathrm{~W}$ and the buoyancy flux is $F_{0}=(P \alpha g) /(c \rho)=5.03 \times 10^{-3} \mathrm{~m}^{4} / \mathrm{s}^{3}$. The surce term in eqn. 3 is given by: $S=H /(c \rho)$

The numerical resolution in the horizontal is $128 \times 128$ grid points, there are 192 grid points in the vertical direction and the time-step is $\Delta t=60 \mathrm{~s}$.

\section{Tilted Plumes}

The surface heat flux of $80 \mathrm{~W} / \mathrm{m}^{2}$ is smaller than the maximum values of heat exchange during specific convection events in the Labrador Sea, Greenland Sea and the Mediteranean (Marshall \& Schott 1999). We express the non alignment of rotation and gravity by giving the latitude $\theta$ of the corresponding situation, that is: $\theta=90^{\circ} \mathrm{N}$ for an alignment of rotation and gravity on the North Pole (NP), $\theta=60^{\circ} N$ for the situation in the Labrador Sea (LS), $\theta=$ $45^{\circ} \mathrm{N}$ for the situation in the Golf of Lions (GL) and $\theta=0^{\circ}$ for the situation at 
the equator (EQ) (rotation being perpendicular to gravity). We are aware of the fact that there is no deep convection at the equator but included the case for completeness of the dynamical picture. We also performed calculations for the case with vanishing rotation (NR) in this case the horizontal expansion of the plume is not arrested, as it is in the rotating case. One-and-a-half days after the onset of cooling the NR-plume's horizontal extension is such that the plume dynamics is strongly influenced by the finite (periodic) domain size (Fig. 1 (a)). In the cases with rotation, the horizontal expansion of the plume is arrested and it only spans a fraction of the horizontal domaine size.

The most conspicuous feature in tilted convection is that the plumes extend in the direction of the axis of rotation rather than gravity (see Fig. 1). This finding, which has been explored by Sheremet 2004 in laboratory experiments for the oceanic context, is well known to researches considering convection in rotating spheres (see e.g. Busse et al., 1998) The elongation of dynamical structures along the axis of rotation is attributed to the Taylor-Proudman Theorem, which states that, in flows dominated by rotation the velocity vector is constant along the axis of rotation.

For the first 5 to 8 hours of the convection experiment the influence of rotation is not visible and all experiments look identical during this time the plume has droped by about 500 meters, being in perfect agrement with the scaling in time $t_{1}=2.4 / \Omega$ and distance $h_{c 1}=3.3\left(F_{0} / \Omega^{3}\right)^{1 / 4}$ proposed by Fernando et al., 1998 (based on laboratory experiments). After that period the plume starts to deviate and follow the axis of rotation. By that time, the rotation inhibits changes of velocity along the axis of rotation (Taylor-Proudman theorem) in the entire domain and the cooled surface water now deviates immediately without an initial vertical sinking. This can be seen in Figs. 1 (c,d), 2 and 3, where two venes of cold water are visible, a lower one formed by the initially vertically convecting and the deviated water, and a upper one formed by the immediately deviating water cooled at later times.

A closer analysis in Figs. 2 and 3, showing a north-south cross-section of the plumes suggests indeed that the plume dynamics is composed of a sinking in the direction of buoyancy force combined with a stretching along the axis of rotation forming "Taylor ink walls" or "Taylor curtains". These Figures compare well to photos of laboratory experiments by Sheremet (2004) and the description in his article. Films composed of snapshots from the numerical data reveal, that the heavier fluid actually spirals along the axis of rotation. The motion does indeed resemble a charged particle in a magnetic field, an analogy that is often advanced. The spiraling motion explains also the eastward drift and the downward drift with respect to the axis of rotation. Indeed, when a buoyant parcel spirals its gravitational acceleration depends on the steepness of the slope, the parcel has thus a faster velocity on the steeper and the following eastward leg, and is slower on the shallower and the following 
westward leg. This leads to a downward and eastward drift of the plume with respect to the axis of rotation (Figs. 2 (b) and 3 (b)), a fact also observed by Sheremet (2004).

After having determined the direction of convection we proceed by looking at the vertical plume speed. After one hour into the experiment and for the following next 7 hours a constant acceleration of the buoyant water front is observed. In Fig. 4 this initial behavior is fitted to a constant acceleration of $g^{\prime}=7.72 \times 10^{-7} \mathrm{~m} / \mathrm{s}^{2}$, this corresponds to a temperature difference of $\Delta T=g^{\prime} /(\alpha g)=3.93 \times 10^{-4} \mathrm{~K}$, which is slightly less than half the maximal temperature after one hour into the experiment. At this early time all five experiments show almost identical behavior as rotation has a vanishing influence. After that initial state the acceleration reduces. This behavior agrees very well with laboratory experiments that showed a decrease of acceleration after $t \approx 2.4 / \Omega$ (Sheremet 2004, Fernando et al., 1998). The plume displacement in the EQ case deviates first from the constant acceleration regime as it exhibits the slowest vertical displacement of all cases, the horizontal rotation vector organizes the plume into horizontal rolls stretched in the north-south direction strongly inhibiting the convection process. In the other extreme case (NP) the rotation only inhibits lateral spreading of the plume making the downward convection process most efficient, as a consequence this case is the last to leave the constant acceleration process of all cases considered. The cases LS and GL are in between these two extremes and more representative of what happens in the ocean. In the early stage the case of no rotation is almost identical to the GL case when the vertical plume displacement is considered, suggesting that for the GL case the retarding effect of the horizontal component of the rotation vector is almost balanced by the accelerating effect of the vertical component of the rotation vector. In other aspects of the dynamics, these two cases are completely different as can be easily seen by comparing Figs. 1 (a) and (d).

In the case of an alignment of rotation and gravity (NP) the behavior agrees well with the laboratory results of Fernando et al. (1998), although our plume is laminar: the plume evolves into a cylindrical fluid column (see Fig. 1) with a radius given by approximately $R_{N P}=1.4\left(F_{0} / \Omega^{3}\right)^{1 / 4}=472 \mathrm{~m}$.

Maxworthy and Narimousa (1994) determined in laboratory experiments that the vertical speed of convective plumes is constant after an initial adjustment and given by:

$$
w=(1 . \pm .1) \sqrt{B / f}
$$

when gravity and rotation vector are aligned and when the buoyancy forcing at the surface is homogeneous, $B$ representing the buoyancy flux per surface area. In our numerical experiments we also determined the vertical plume dis- 
placement after the initial adjustment. The results are given in Fig. 5 (a). After about 6 to 8 hours the vertical plume displacement proceeds at almost constant velocity, except for the case with no rotation and the equatorial case. The magnitude of the velocity is also in good agreement with eq. (6). Deviations from the constant velocity regime are also observed some three-hundred meters before the plume reaches the floor, a length scale that corresponds to the horizontal dimension of the plume. This comes at no surprise as in flows dominated by rotation, the variation of the velocity vector along the axis of rotation is inhibited (Taylor-Proudman theorem). In Fig. 5 (b) the displacement of the plume is divided by the sine of the latitude and it is clearly visible that the renormalized vertical velocity agrees for all three cases considered. Based on this findings we can thus extent the formula (6) of Maxworthy and Narimousa (1994) to cases of tilted convection and obtain:

$$
w=\sqrt{B / f} \sin (\theta)=\sqrt{\frac{B \sin (\theta)^{3}}{f_{\perp}}},
$$

where $f=2 \Omega, f_{\perp}=2 \Omega \sin (\theta)$. The best fit for the normalized velocities gives $w\left(\theta=0^{o}\right)=2.25 \times 10^{-2} \mathrm{~m} / \mathrm{s}$.

The plume dynamics at the equator (EQ) is different from all other cases in many aspects. No coherent plume is formed the heavy water sinks and spirals around the horizontal axis of rotation extending in the north-south direction. The extension in this direction is not inhibited as the vertical component of the rotation vector vanishes. No coherent plume speed can be obtained in this case.

Another important point is the increased mixing in tilted convection already mentioned in Sheremet (2004). Figures 1 (c,d and e) show indeed smaller size features than Fig. 1 (b) which shows a single coherent axis-symmetric structure. In the case of tilted convection the axis-symmetry is broken and the plume evolves in a more chaotic way. The presence of the fine structures as for example thin "Taylor ink walls" in the cases LS and GL increase the mixing as compared to the NP or NR case. The horizontally averaged temperature one-and-a-half days into the convection experiment are shown in Fig. 6. It can be clearly seen that in the experiments involving a vertical and a horizontal component of the rotation axis the mean temperature at each level is decreased (more negative temperature!) after the passage of the plume head as compared to the case with a vertical axis of rotation, the average temperature in the NR and NP cases is only about half of the value in the LS and GL cases. In the case corresponding to the Labrador Sea and the Golf of Lions the water column above the tip of the front is almost perfectly homogenized. 


\section{Conclusions and Perspectives}

Based on direct numerical simulations, we have demonstrated that the horizontal component of the rotation vector has significant qualitative and quantitative consequences on the dynamics of a single convective plume at timescales longer than about a quarter of a day. Our here presented numerical calculations are in close agreement with laboratory experiments of Sheremet (2004). The convection is composed of a sinking in the direction of buoyancy force combined with a stretching along the axis of rotation. The vertical speed of the plume is reduced by the horizontal component of the rotation, and the mixing is increased. We furthermore extent an existing formula for the vertical plume speed in the case of aligned gravity and rotation vectors to the case of an arbitrary angle.

The here presented case was restricted to the dynamics of a single plume in an isothermal ocean leading to deterministic convection laws. At convection sites stratification is generally weak as the the water masses are well mixed by previously occured convection events and, as the restratification process is slow (seasonal time scale). The analysis of the important case of convection by an ensemble of plumes in an isothermal and stably stratified ocean is of stochastic nature. Such investigation, possibly leading to improved parameterizations of open ocean convection, is presently in progress. An improved parameterization

of convection in the Greenland Sea $\left(75^{\circ} \mathrm{N}\right)$, Labrador Sea $\left(60^{\circ} \mathrm{N}\right)$ and the Golf of Lions $\left(45^{\circ} \mathrm{N}\right)$, including "non-traditional" effects, is likely a key to improving hydrostatic ocean global circulation models (OGCMs), especially when problems on climate time-scales are considered.

\section{References}

[1] F. H. Busse, G. Hartung, M. Jaletzky \& G. Sommermann (1998), Experiments on thermal convection in rotating systems motivated by by planetary problems. Dyn. Atmos. Oceans 27, 161-174.

[2] H.J.S. Fernando, R-r Chen, and A. Ayotte (1998) Development of a point plume in the presence of background rotation, Phys. Fluids, 10, pp. 2369-2383.

[3] D. Goldstein, R. Handler, and L. Sirovich (1993) Modeling a no-slip flow boundary with an external force field, J. Comp. Phys.. 105, pp.354-366. 
[4] H. Jones and J. Marshall (1993) Convection with rotation in a neutral ocean: a study of open-ocean deep convection, J. Phys. Oceanogr., 23, pp.1009-1039.

[5] P. D. Killworth (1983), Deep convection in the world ocean, Rev. Geophys., 21, pp. 1-26.

[6] J. Marshall, and F. Schott, Open-ocean convection: observations, theory, and models, Rev. Geophys., 37, pp. 1-64.

[7] J. Marshall, C. Hill, L. Perelman and A. Adcroft (1997) Hydrostatic, quasihydrostatic, and nonhydrostatic ocean modeling, J. Phys. Oceanogr., 27, pp. $5733-5752$.

[8] T. Maxworthy and S. Narimousa (1994) Unsteady, turbulent convection into a homogeneous, rotating fluid, with oceanographic applications, J. Phys. Oceanogr., 24, pp. 865-887.

[9] C. S. Peskin (1977) Flow patterns around heart valves: a numerical method, J. Comp. Phys.. 25, pp. 220-252.

[10] F. Schott and K. D. Leaman (1991) Observation with moored acoustic Doppler current profiles in the convection regime in the Golf de Lion. J. Phys. Oceanogr., 21, pp.558-574.

[11] F. Schott, M. Visbeck and J. Fischer (1977) Observations of vertical currents and convection in the Central Greenland Sea during the winter of 1988-1989, J. Geophys. Res.. 98, pp. 14 401-21.

[12] U. Send \& J. Marshall (1995) Integral effects of deep convection, J. Phys. Oceanogr., 25, pp.885-872.

[13] V. Sheremet (2004) Laboratory experiments with tilted convective plumes on a centrifuge: a finite angle between the buoyancy force an the axis of rotation. J. of Fluid Mech. 506, pp. 217-244.

[14] J. Willebrand, B. Barnier, C. Bönning, C. Dietrich, P.D. Killworth, C. LeProvost, Y. Yia,J.-M. Molines, A.L. New (2000), Circulation characteristics in three eddy-permitting models of the North Atlantic. Progr. in Oceanogr. 48, $123-161$.

[15] A. Wirth (2004), A non-hydrostatic flat-bottom ocean model entirely based on Fourier Expansion, Ocean Mod., 9, 71-87, 2004.

[16] R.A. Wood, A.B. Keen, J.F.B. Mitchell, et J.M. Gregory (1999), Changing the spatial structure of the thermohaline circulation in response to atmospheric $\mathrm{CO}_{2}$ forcing in a climate model. Nature 372, pp. 572-575. 


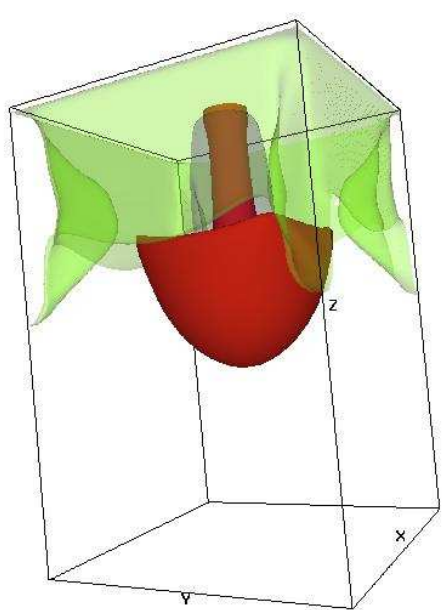

(a)

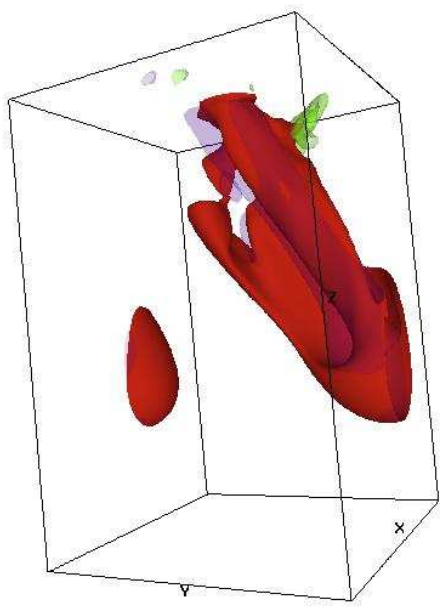

(c)

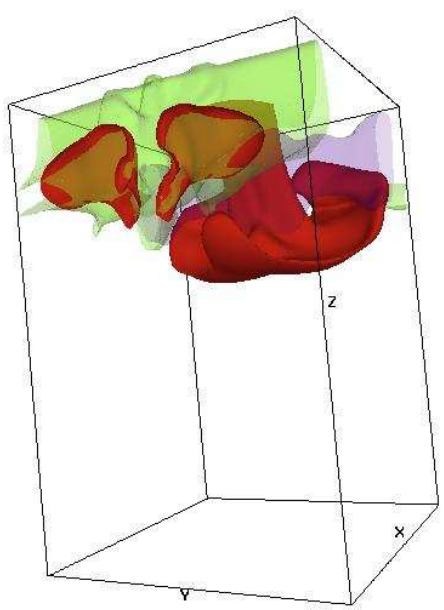

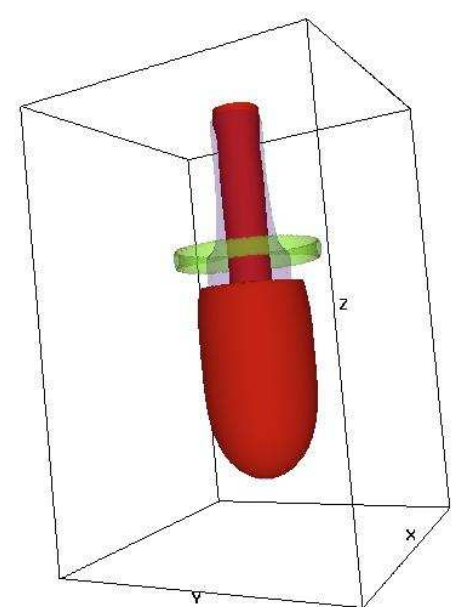

(b)

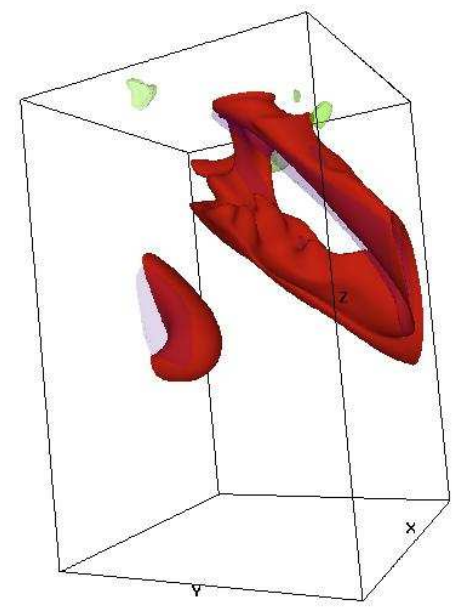

(d)

(e)

Fig. 1. Temperature isosurface $\Delta T=5 . \times 10^{-4} \mathrm{~K}$ (red) and vertical velocity isosurfaces $w=-.01 \mathrm{~m} / \mathrm{s}$ (violet) and $w=.01 \mathrm{~m} / \mathrm{s}$ (green) at $t=1.5$ days after the onset of cooling, for different latitudes on earth (a) NR (no rotation), (b) NP $\left(90^{\circ} \mathrm{N}\right)$, (c) LS $\left(60^{\circ} \mathrm{N}\right),(\mathrm{d}) \mathrm{GL}\left(45^{\circ} \mathrm{N}\right)$ and $(\mathrm{e}) \mathrm{EQ}\left(0^{\circ} \mathrm{N}\right)$. 

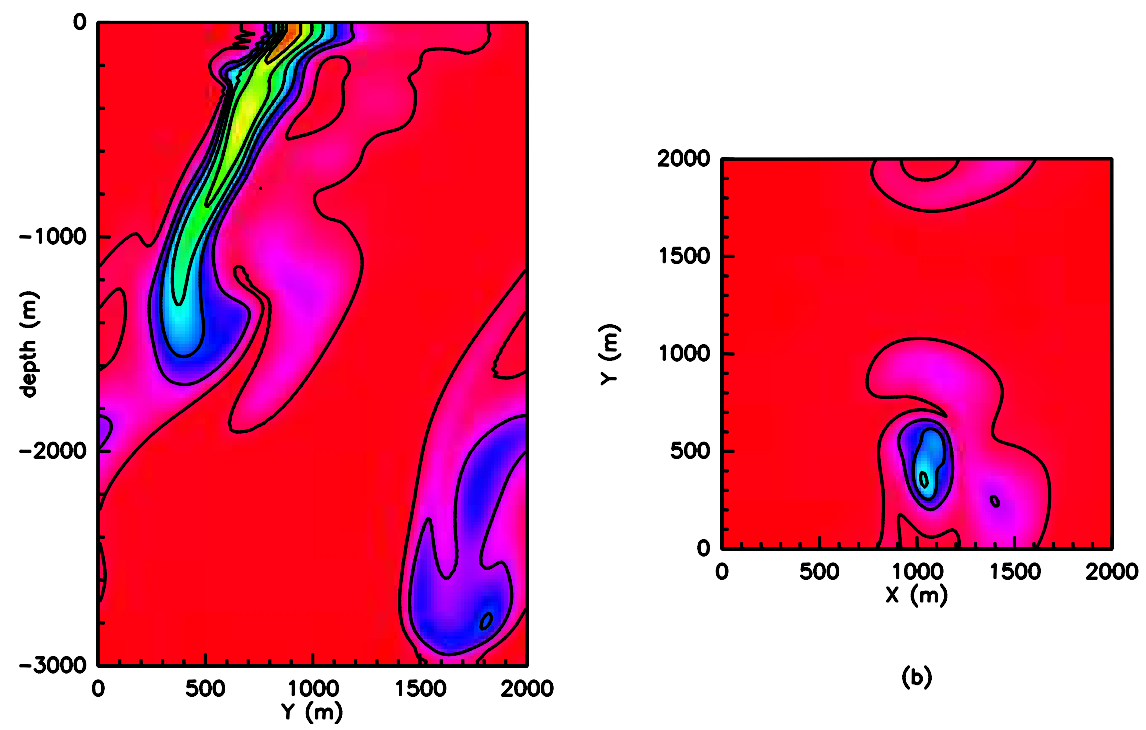

(a)

Fig. 2. LS case $\left(\theta=60^{\circ} N\right)$; (a) South-North cut through the center of the domain and (b) horizontal cut at $1500 \mathrm{~m}$ depth, temperature contour lines every $\Delta T=5 . \times 10^{-4} \mathrm{~K}$ starting from $\Delta T=3.1 \times 10^{-3} \mathrm{~K}$ at $t=2$ days after the onset of cooling.
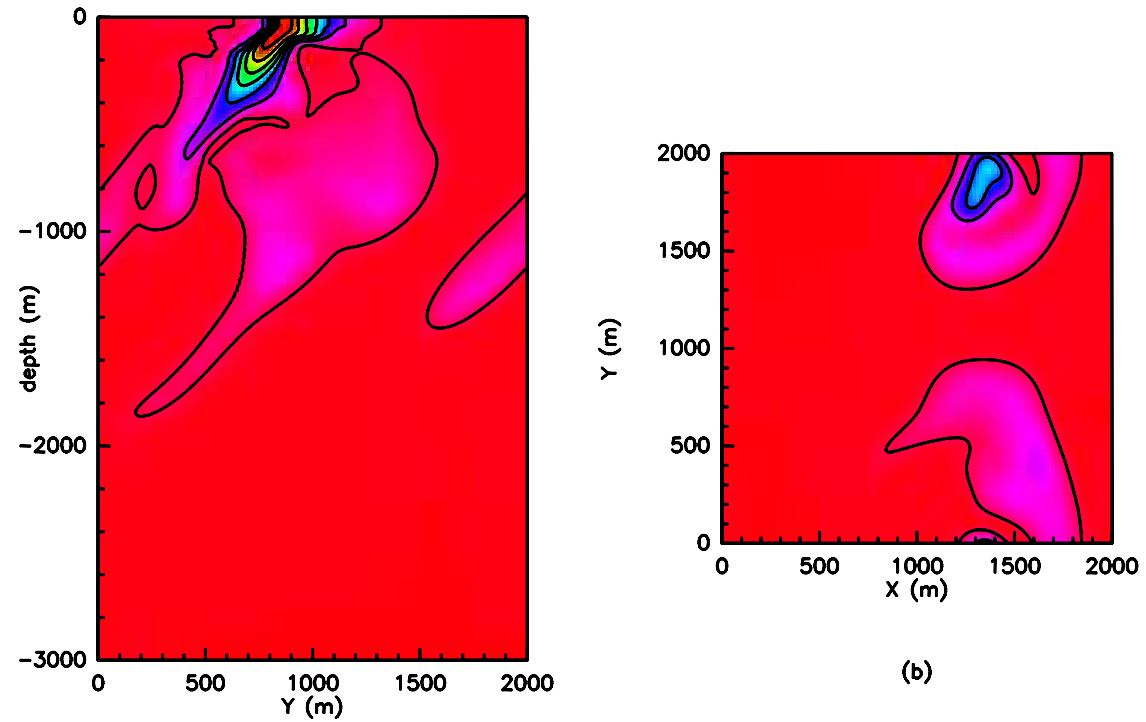

(a)

Fig. 3. GL case $\left(\theta=45^{\circ} N\right)$; (a) South-North cut through the center of the domain and (b) horizontal cut at $1500 \mathrm{~m}$ depth, temperature contour lines every $\Delta T=5 . \times 10^{-4} \mathrm{~K}$ starting from $\Delta T=3.1 \times 10^{-3} \mathrm{~K}$ at $t=2$ days after the onset of cooling. 


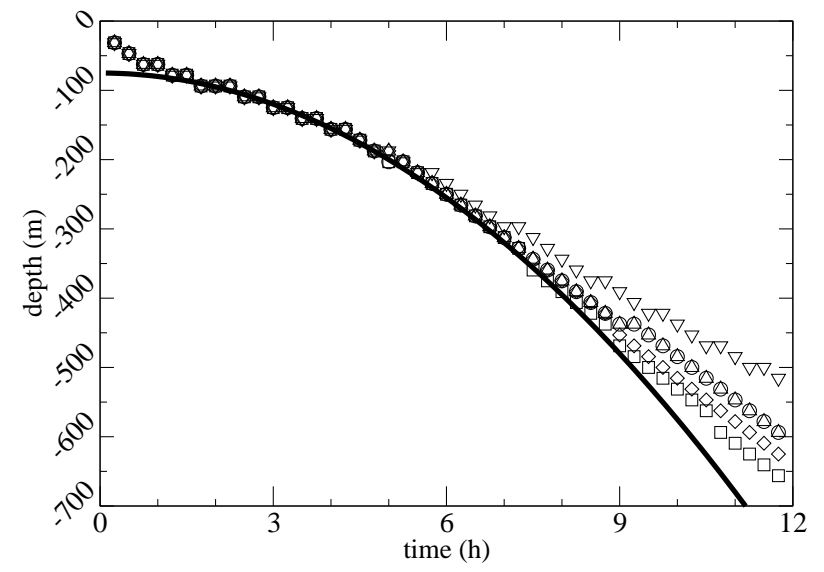

Fig. 4. Vertical displacement of plumes for the first 12 hours. (-) Fit (const. acceleration); $(\square) \mathrm{NP} ;(\diamond) \mathrm{LS} ;(\triangle) \mathrm{GL} ;(\nabla) \mathrm{EG} ;(\bigcirc) \mathrm{NR}$
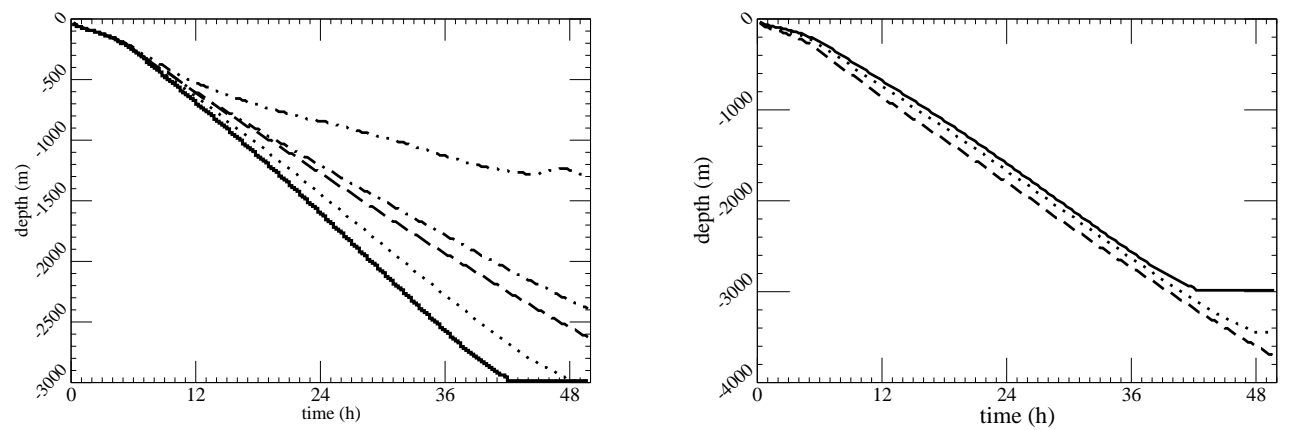

Fig. 5. Vertical displacement of plumes (a) and vertical displacement of plumes divided by the sine of the latitude (b). (-) NP, (..) LS, (- - -) GL, (- . - ) NR and $(-\cdot-\cdot \cdot)$ EQ. 


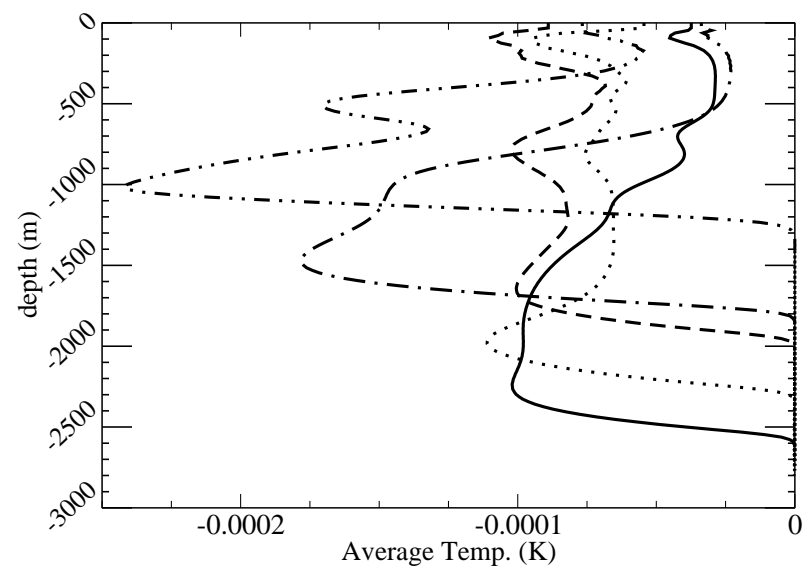

Fig. 6. Horizontally averaged temperature 1.5 days into the experiment. (-) NP, $(\cdots)$ LS, $(---)$ GL, $(-\cdot-\cdot)$ NR and $(-\cdot-\cdot \cdot)$ EQ. 